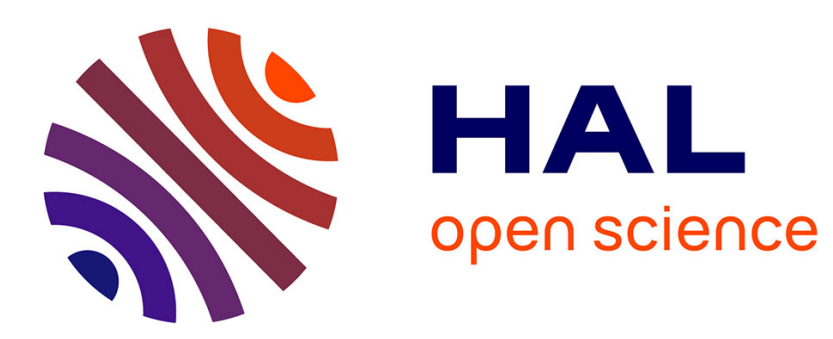

\title{
Datation absolue des cuvettes à pierres chauffées de Claireaux à Cubord, Valdivienne (Vienne)
}

\author{
Jean-Pierre Pautreau, Michel Fontugne
}

\section{To cite this version:}

Jean-Pierre Pautreau, Michel Fontugne. Datation absolue des cuvettes à pierres chauffées de Claireaux à Cubord, Valdivienne (Vienne). Bulletin de la Société préhistorique française, 1996, 93 (4), pp.543 545. 10.3406/bspf.1996.10216 . hal-03545391

\section{HAL Id: hal-03545391 https://hal.science/hal-03545391}

Submitted on 27 Jan 2022

HAL is a multi-disciplinary open access archive for the deposit and dissemination of scientific research documents, whether they are published or not. The documents may come from teaching and research institutions in France or abroad, or from public or private research centers.
L'archive ouverte pluridisciplinaire HAL, est destinée au dépôt et à la diffusion de documents scientifiques de niveau recherche, publiés ou non, émanant des établissements d'enseignement et de recherche français ou étrangers, des laboratoires publics ou privés. 


\section{Datation absolue des cuvettes à pierres chauffées de Claireaux à Cubord, Valdivienne (Vienne) Jean-Pierre Pautreau, Michel Fontugne}

\section{Résumé}

RESUME Dans la vallée de la Vienne, l'ensemble des cuvettes à pierres chauffées des Claireaux à Valdivienne (Vienne) comportait au moins 6 structures. La datation absolue des charbons de l'une d'elles permet une attribution au Néolithique moyen.

\section{Abstract}

ABSTRACT Located in the Vienne river valley, Claireaux (Valdivienne, Vienne) is a site with six burned stone cooking pits. The UC date obtained from charcoal from a cooking pit permits dating to the Middle Neolithic.

\section{Citer ce document / Cite this document :}

Pautreau Jean-Pierre, Fontugne Michel. Datation absolue des cuvettes à pierres chauffées de Claireaux à Cubord, Valdivienne (Vienne). In: Bulletin de la Société préhistorique française, tome 93, n4, 1996. pp. 543-545;

doi : https://doi.org/10.3406/bspf.1996.10216

https://www.persee.fr/doc/bspf_0249-7638_1996_num_93_4_10216

Fichier pdf généré le 10/01/2019 


\title{
DATATION ABSOLUE DES CUVETTES A PIERRES CHAUFFÉES DES CLAIREAUX A CUBORD, VALDIVIENNE (VIENNE)
}

\author{
Jean-Pierre PAUTREAU et Michel FONTUGNE
}

\section{RÉSUMÉ}

Dans la vallée de la Vienne, l'ensemble des cuvettes à pierres chauffées des Claireaux à Valdivienne Nienne) comportait au moins 6 structures. La datation absolue des charbons de l'une d'elles permet une attribution au Néolithique moyen.

\section{ABSTRACT}

Located in the Vienne river valley, Claireaux (Valdivienne, Vienne) is a site with six burned stone cooking pits. The ${ }^{14} \mathrm{C}$ date obtained from charcoal from a cooking pit permits dating to the Middle Neolithic.

Lors des travaux de sauvetage effectués au printemps 1986 dans le cadre de l'édification de la centrale nucléaire de Civaux (Vienne), plusieurs groupes de cuvettes à pierres chauffées ont pu être mis en évidence. L'ensemble le plus remarquable se trouve sur la terrasse de la rive gauche, à proximité du village des Claireaux, non loin de la confluence de la Vienne avec la Dive de Morthemer, à $500 \mathrm{~m}$ du lit de la rivière. II comprenait les traces d'au moins 6 structures de combustion allongées mesurant 7 $\mathrm{m}$ à $9 \mathrm{~m}$ de long et de structures circulaires ou sub-ovalaires (fig. 1). Ces foyers, sensiblement orientés sud-est - nord-ouest contiennent au milieu d'un sédiment charbonneux bien noir des quantités de galets éclatés au feu (fig. 2 et 3). Certains semblaient associés à des trous de poteau et à des fosses mais l'absence d'artefact caractéristique et la présence d'une nécropole médiévale perturbatrice de l'ensemble préhistorique ne permettent pas d'affirmer ces liens. Les cuvettes à pierres chauffées des Claireaux ont déjà été décrites (Pautreau, Mataro i Pladelasala et Villard, 1993 ; Pautreau et Mataro i Pladelasala, 1987 , p. 300 , fig. 7 et 8 ; Pautreau et Mataro i Pladelasala, 1988, p. 73 ; Mataro i Pladelasala et Pautreau - in : Joussaume et Pautreau, 1990, p. 165166 ; Mataro i Pladelasala et Pautreau,

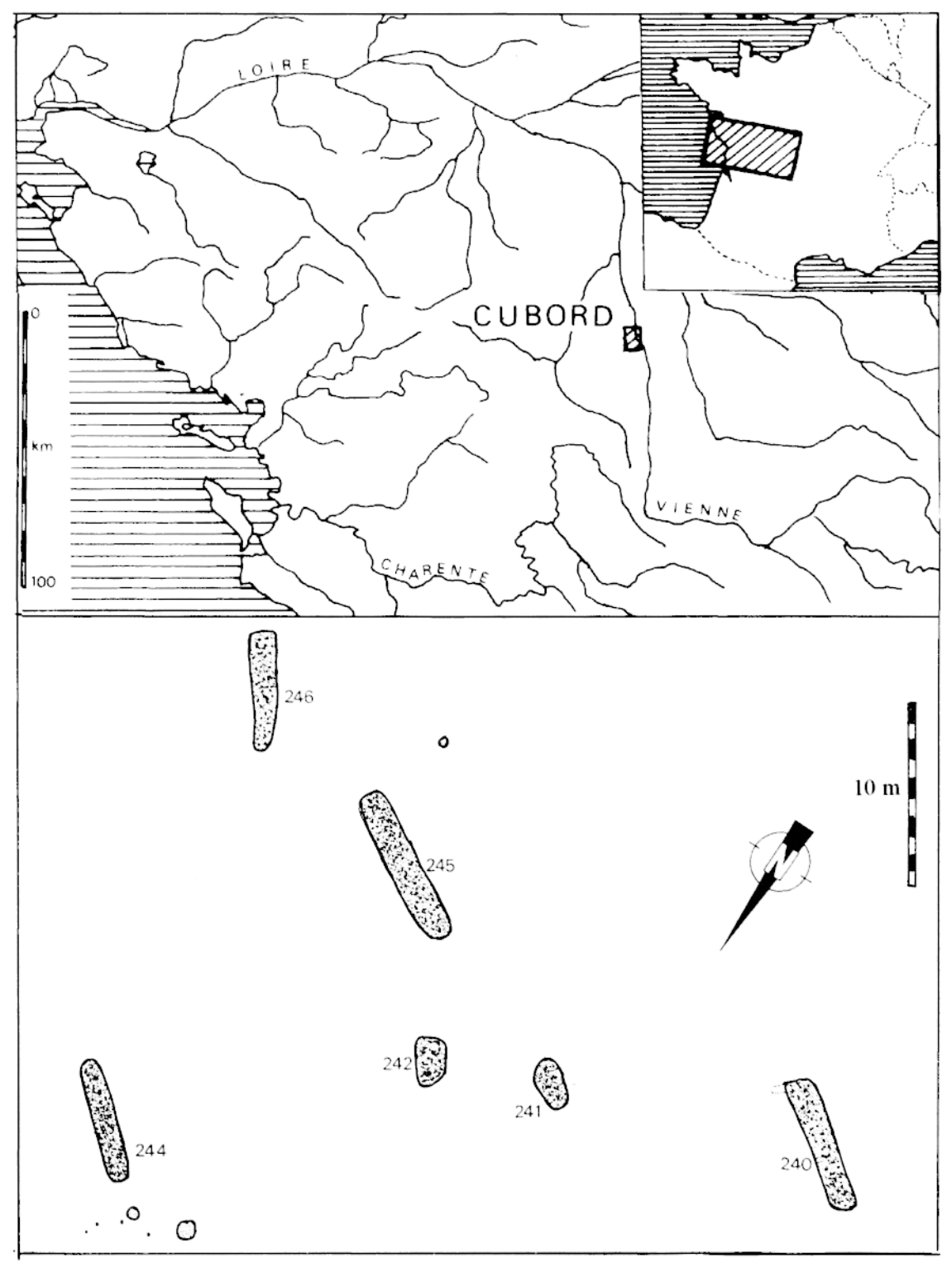

Fig. 1 - Les Claireaux, Valdivienne (Vienne). Situation (Relevé M. Mataro i Pladelasala).

in : Boissavit-Camus, Papinot et Pautreau 1991, p. 48).

Plusieurs caractéristiques apparaissent constantes au sein de ces structures de blocs éclatés. Dans tous les cas l'aménagement des pierres dans une cuvette plus ou moins allongée est de règle. La grosseur moyenne des blocs $(30 \mathrm{~cm} / 40 \mathrm{~cm})$ varie peu. A l'exception des rares calcaires, ils proviennent tous de la terrasse; leur quête a fait l'objet d'une longue recherche ; les galets de quartz, micaschiste et granite d'une telle taille, sans être exceptionnels demeurent rares. Dans tous les cas, ces galets disposés en une ou deux couches, ont été chauffés in situ, ont éclaté sur place et n'ont subi aucun déplacement ultérieur. Leur contact avec le feu semble se faire sur toutes les faces. Les déchets de combustion apparaissent mieux conservés à la base 


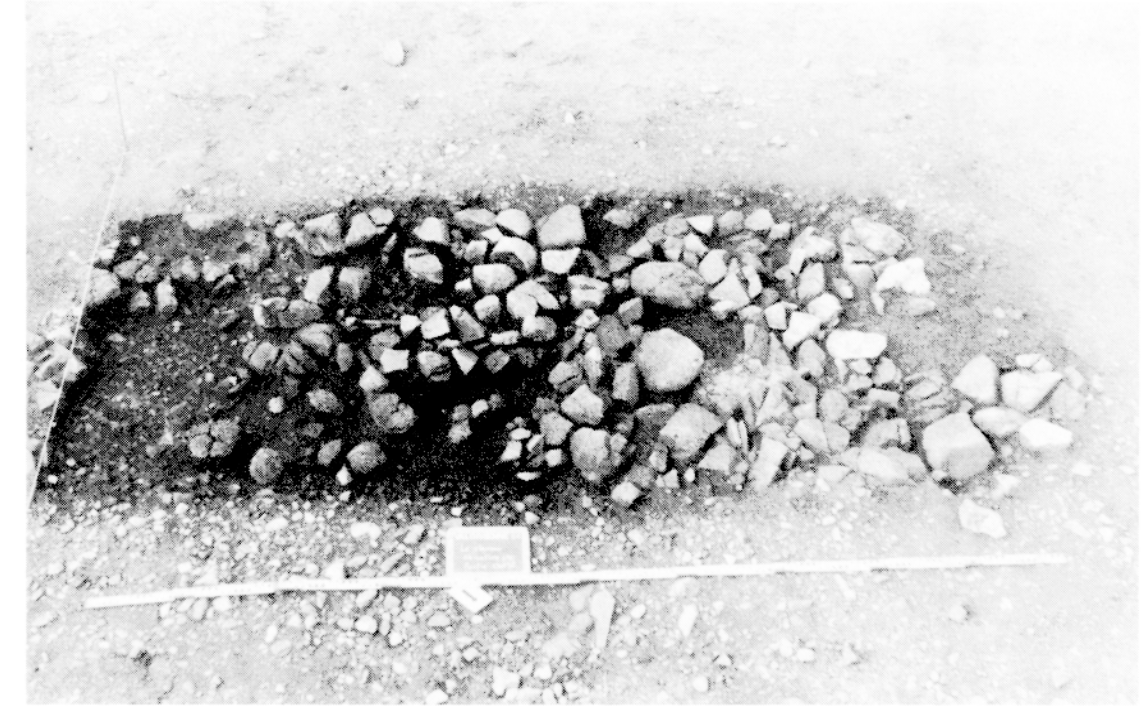

Fig. 2 - Les Claireaux, Valdivienne (Vienne). Structure de combustion allongée $n^{\circ}$ 240. Extrémité nord-ouest (Photographie S. Vacher).

et dessous les gros galets. II semble manifeste que les galets n'ont jamais été en contact avec le fond ou les bords souvent rubéfiés des cuvettes.

Il est probable qu'après le creusement de la cuvette. du bois (traces de branchages) était déposé au fond : c'est semble-t-il postérieurement que furent installées les pierres ; ensuite seulement des rondins ou gros branchages disposés sur les blocs et fort soigneusement et régulièrement entre ceux-ci et les bords, formant une couverture végétale (sorte de toit à double pente ?) recouvraient la nourriture à cuire. Le brasier ne devait être allumé que dans une dernière phase. II est possible aussi que les gros galets aient èté déposés sur des braises encore brûlantes : cette hypothèse, plus convaincante sur le plan de la combustion n'est pas entièrement satisfaisante, ne permettant pas d'expliquer l'auréole parfois large et bien régulière de charbon séparant les pierres des bords de la cuvette.

C'est dans le bassin de la Garonne que l'on trouve le plus grand nombre de structures en cuvette à galets chauffés permettant des comparaisons. L'ensemble des Claireaux n'atteint pas la densité des gisements chasséens de VilleneuveTolosane (Clottes et coll., 1980, p. 6 : Clottes et coll., 1981, p. 116), de Frouzins (Clottes et coll., 1977 , p. 583), de Château-Percin (Vaquer, 1990, p. 217) ou de St-Michel-duTouch (Simonet, 1976, p. 15 ; Simonet, 1980, p. 951, Giraud et Vaquer. 1981, p. 36) près de Toulouse. Son

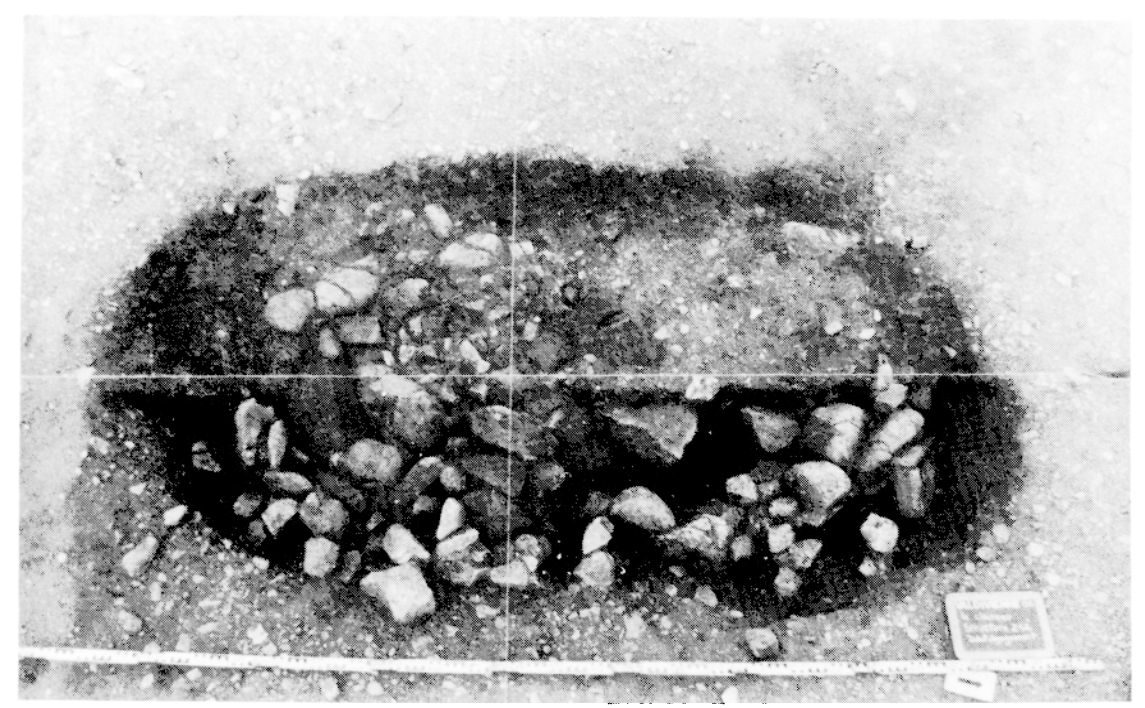

Fig. 3 - Les Claireaux, Valdivienne (Vienne). Structure de combustion courte $n^{\circ} 242$. Vue générale (Photographie $S$. Vacher). organisation comme sa morphologie le rapprochent plutôt des gisements à cuvettes de galets chauffés à petits nombre de structures comme ceux de Carbonne et de Beauzelle toujours en Haute-Garonne (Giraud et Vaquer, 1981, p. 38-39).

De telles cuvettes garnies de galets chauffés, rubéfiés et éclatés restent rares en France en dehors du bassin de la Garonne où elles furent pour la première fois mises en évidence. Pour les expliquer, on a tour à tour parlé de sols de cabanes, d'ateliers de boucanage, de fours polynésiens, de foyers rituels, etc. Une bonne mise au point vient d'être publiée (Vaquer, 1990, p. 296-300). Toute explication définitive demeure impossible ; aux Claireaux, les esquilles d'os, fort rares au demeurant (une épiphyse éclatée et indéterminable dans la structure $n^{\circ} 240$ ), invitent à envisager un rôle culinaire pour ces imposants aménagements dont le fonctionnement exact nous échappe. De récentes études sur les foyers et structures de combustion préhistoriques (Beeching et Gasco, 1989, p. 275-292 ; Audouze, 1989, p. 327-334; Pons et Molist, 1989, p. 137-142) ne permettent pas de préciser l'utilisation de ces constructions en dehors des normes généralement admises pour les foyers domestiques.

Les vestiges archéologiques associés aux cuvettes des Claireaux apparaissent limités. Un tesson orné d'impressions ongulées découvert dans le niveau supérieur de la structure $n^{\circ} 240$ reste peu caractéristique dans la cuvette $n^{\circ} 241$, au sommet des quartzites, micaschistes et granites brûlés, se trouvait un fragment informe de céramique non tournée. Un éclat de silex a été mis au jour au fond de la fosse $n^{\circ} 242$ (fig. 3). Une petite cuvette circulaire $\left(n^{\circ} 243\right)$ recelait quelques vestiges céramiques, lithiques et des esquilles osseuses calcinées au sein de zones charbonneuses et cendreuses diffuses, sans galets éclatés. L'attribution de cette dernière fosse au Néolithique moyen paraît fort envisageable, au regard notamment de la céramique (anse tunnelée appliquée à léger renflement interne, fragments d'écuelles), mais aucun vestige ne permet de proposer un ensemble culturel précis.

Dans les remplissages, sur et sous les blocs, plusieurs fragments de bois carbonisés ont été préservés. Un rondin, retrouvé dans l'axe 
de la structure $n^{\circ} 245$. a pu être suivi sur près de $50 \mathrm{~cm}$ de long ; il mesurait plus de $15 \mathrm{~cm}$ de diamètre.

Les datations ont été effectuées sur des charbons prélevés dans la fosse de combustion $n^{\circ} 246$, cuvette allongée mesurant $6,60 \mathrm{~m}$ de long et 1,10 à $1,30 \mathrm{~m}$ de large (fig. 1). Les charbons analysés proviennent du lit charbonneux reposant entre la dernière couche de blocs éclatés et les parois par endroits rubéfiées.

Les résultats obtenus sont les suivants :

GIF 10030 Cubord Les Claireaux Bois $n^{\circ} 3$.

$5420 \pm 100$ ans soit en date calibrée : Cal BC $(4455,4003)$.

Intervalle de confiance $95 \%$ (2 sigmas).

Calibration d'après Stuiver et Reimer, 1993 (Rad v 35, $n^{\circ}$ 1, 1993 : p. 215-230)

GIF 10031 Cubord Les Claireaux Bois $n^{\circ} 4$.

$5580 \pm 50$ ans soit en date calibrée: Cal BC $(4504,4340)$.

Intervalle de confiance $95 \%$ (2 sigmas).

Calibration d'après Stuiver et Reimer, 1993 (Rad v 35, n 1, 1993, p. 215-230).

Les fourchettes de dates proposées correspondent, dans le CentreOuest, au Néolithique moyen. Dans la même vallée de la Vienne, deux kilomètres plus au sud, le site du Grand-Champ à Gavid a livré au milieu de vestiges en creux d'époques diverses, plusieurs fosses allongées du type "four polynésien " mais qui n'ont pas produit une quantité de charbon suffisante pour permettre une datation. Toutefois, à proximité, deux petites fosses circulaires aux parois rubéfiées semblent devoir être interprètées comme des fours (Pautreau et Mornais, 1994). La datation des charbons de bois prélevés au fond d'une de ces cavités, réalisée par M. Fontugne, a donné la mesure de $5220 \pm 80$ ans (Gif-9557), soit en date calibrée Cal BC $(4240,3806)$, correspondant là encore au Néolithique moyen. Dans la région proche les vestiges du Néolithique moyen se limitent à des mobiliers funéraires. Les tombes en fosse d'Antran semblent attribuables à un faciès antérieur aux utilisateurs de coupes à socles rencontrés dans les sépultures mégalithiques de Busserais à la Bussière et de la Bassetière à Sillars. Quelques enceintes : la CroixBlanche d'Antran et à la Rabatelle de Lésigny sont à rapprocher, avec leur plan sub-ovalaire et leur petit nombre d'interruptions, d'enceintes chasséennes du Bassin Parisien. II reste impossible de rattacher les structures de combustion des Claireaux à un groupe culturel précis.

\section{Bibliographie}

Audouze F. (1989) - Foyers et structures de combustion domestiques aux âges des métaux. Nature et fonction des foyers préhistoriques, actes du Colloque de Nemours, 1987. Mémoires du Musée de Préhistoire d'ille-deFrance, 2, p. 327-334, 5 fig.

BeEching A. et Gasco J. (1989) - Les foyers de la Préhistoire récente du Sud de la France (Descriptions, analyses et essais d'interprétation). Nature et fonction des foyers préhistoriques. actes du Colloque de Nemours. 1987. Mémoires du Musée de Prèhistoire d'île-de-France, 2, p. 275-291, 16 fig.

Boissavit-Camus B., Papinot J.-C. et PauTREAU J.-P. (1991) - Civaux. Des origines au Moyen-Age. Mémoire horssérie, sous l'égide d'E.D.F., Assoc. des Publications Chauvinoises, $119 \mathrm{p}$.

Clottes J.. Giraud J.-P.. Rouzaud F. et VAQUER J. (1981) - Le village chasséen de Villeneuve Tolosane (HauteGaronne). Fouilles 1978, C.P.F. XXI' session, Quercy. 1979. 1. p. 116-128. $10 \mathrm{fig}$.

Clottes J.. Giraud J.-P. el Vaquer J. (1979) - Le village néolithique de Villeneuve Tolosane. Archéologia. $n=130$, p. 6-13, 8 fig.

Clottes J Querre J., Rouzaud F. et Sarny H. (1977) - Les structures chasséennes de Frouzins (Haute-Garonne), B.S.P.F., t. 74, 2. p. 583-607, 19 fig.
GIRAUD J.-P. et VAQUeR J. (1981) - Nouvelles structures à galets chauffés dans la Haute-Garonne. Bull. de la Soc. Méridionale de Spéléologie et de Préhistoire, XXI, p. 35-50. 9 fig.

Joussaume R. el Pautreau J.-P. (1990) La Préhistoire du Poitou. Ed. OuestFrance Universitè. 599 p.

Pautreau J.-P. et Mataro I Pladelasala M. (1990) - Les enclos protohistoriques de Cubord à Valdivienne (Vienne). Étude Préliminaire. Préhistoire de Poitou-Charentes, Problèmes actue/s, 111" Cong. Nat. des Soc. Sav.. Poitiers, Prè et Protohistoire, p. 289-306, 8 fig.

Pautreau J.-P., Mataro I Pladelasala M., VILLARD A. et coll. (1993) - Civaux Valdivienne II. Les nécropoles protohistoriques et structures néolithiques : Enclos, fosses, structures de combustion. Socièté de Recherches Archéologiques et Historiques du Pays Chauvinois, mémoire VII. 168 p..153 fig., ph. h. $-t$.

Pautreau J.-P. et Mornais P. (1994) Deux structures de combustion du Néolithique moyen au Grand-Champ à Gavid, Valdivienne (Vienne), XXI" Colloque interrégional sur le Néolithique, Poitiers. 14-16 octobre 1994, résumés. p. 19-20.

PONS E. et MOLIST M. (1989) - Les structures domestiques de cuisson durant la Protohistoire en Catalogne. Habitats et structures domestiques en Méditerranée occidentale durant la Protohistoire. Colloque intern. Arles-surRhône, 19-21 oct. 1989, pré-actes. p. $137-142,2$ fig.

SIMONET G. (1976) - Le village chasséen de Saint-Michel du Touch à Toulouse (Haute-Garonne), IX Congrès UISPP. Livret-guide. excursion A5. Pyrénées. p. 15-34, 15 fig.

SIMONET G. (1980) - La structure chasséenne "V.T. 215 " à Villeneuve Tolosane (Haute-Garonne), B.S.P.F.. t. 77. 5. p. 144-151, 7 fig.

VAQUER J. (1990) - Le Néolithique du Languedoc occidental. Paris, C.N.R.S. 398 p.. 7 pl.

Jean-Pierre PAUTREAU C.N.R.S. U.M.R. 153 Laboratoire d'Anthropologie Université de Rennes 1

Michel FONTUGNE Centre des Faibles radioactivités Laboratoire mixte C.N.R.S. - C.E.A Gif-sur-Yvette 